\title{
Comunidade de vespas sociais (Hymenoptera, Vespidae) em diferentes gradientes altitudinais no sul do estado de Minas Gerais, Brasil
}

\author{
Carlos Humberto Bueno de Albuquerque ${ }^{1}$ \\ Marcos Magalhães de Souza ${ }^{1 *}$ \\ Mateus Aparecido Clemente ${ }^{2}$ \\ ${ }^{1}$ Instituto Federal do Sul de Minas, Campus Inconfidentes \\ Praça Tiradentes, 416, Centro, CEP 37576-000, Inconfidentes - MG, Brasil \\ ${ }^{2}$ Universidade Estadual Paulista, Campus de Rio Claro \\ Avenida 24 A, 1515, Bela Vista, CEP 13506-900, Rio Claro - SP, Brasil \\ * Autor para correspondência \\ marcos.souza@ifsuldeminas.edu.br
}

Submetido em 06/04/2015

Aceito para publicação em 26/09/2015

\section{Resumo}

Vários estudos descreveram alterações na riqueza e abundância de grupos de insetos ao longo dos gradientes altitudinais, contudo há poucas informações para as vespas sociais (Hymenoptera, Vespidae). O presente trabalho avaliou a influência da altitude sobre a diversidade desses insetos em fragmentos de florestas estacionais semideciduais em clima tropical de altitude. $\mathrm{O}$ estudo foi conduzido em três cotas altimétricas distintas na região sul do estado de Minas Gerais, entre 997 e 2080 m. Foram realizados 24 dias de amostragem durante dez meses por meio de coleta ativa. Foram registradas 34 espécies distribuídas em 11 gêneros, a Cota 01 registrou 27, a Cota 02 registrou 19 espécies e a Cota 03 foram registradas 11 espécies. O gênero mais frequente na área de estudo foi Polybia. O trabalho mostra que em áreas de clima tropical de altitude acima de $1800 \mathrm{~m}$ a diversidade diminui consideravelmente, e aumenta o número de espécies que nidificam por enxameamento e constroem ninhos com invólucro protetor.

Palavras-chave: Biogeografia; Fragmentos florestais; Insetos; Polistinae

\section{Abstract}

Social wasp communities (Hymenoptera Vespidae) at different altitudinal levels in southern Minas Gerais, Brazil. Various studies have described changes in richness and abundance of insect groups along altitudinal gradients, but there is little information about social wasps (Hymenoptera, Vespidae) in this regard. This study evaluated the influence of altitude on the diversity of these insects in fragments of semideciduous forest in a tropical altitude climate. Three different elevations in southern Minas Gerais, from 997 to 2080 meters, were studied. Sampling was carried out on 24 days in a ten-month period by active collection. A total of 34 species were found, distributed in 11 genera. At Level 01, 27 species were recorded, and at Level 02, there were 19 species, while Level 03 showed 11 species. The most frequent genus in the sudy area was Polybia, This study showed that in high-altitude tropical climate areas above 1800 meters, diversity decreases substantially and that the number of species that nest by swarming and build nests with protective cover increases.

Key words: Biogeography; Forest fragments; Insects; Polistinae 


\section{Introdução}

As variações ocasionadas pelos gradientes de altitude afetam a distribuição, biodiversidade, bem como abundância e frequência dos organismos (JANZEN, 1973; WOLDA, 1987). Essas variações altitudinais ainda representam uma das grandes fronteiras biogeográficas a serem estudadas que pode auxiliar no entendimento das respostas dos organismos às mudanças ambientais (HENRIQUES-OLIVEIRA; NESSIMIAN, 2010).

Vários estudos descreveram alterações na riqueza e abundância de grupos de insetos ao longo de gradientes altitudinais. O padrão geral encontrado é a diminuição da riqueza de espécies com o aumento da altitude (JANZEN, 1973; WOLDA, 1987; FERNANDES; LARA, 1993; SANDERS, 2002; KUMAR et al., 2009). Essa diminuição pode estar relacionada a reflexos do aumento da severidade climática, redução da área de habitat, diversidade de recursos e produtividade primária (LAWTON et al., 1987; FERNANDES; PRICE, 1991), além de fatores morfofisiológicos adaptativos, que estariam ausentes ou mais raros nas espécies de altitudes mais baixas (JANZEN, 1973; O'DONNELL, 2000; KUMAR, et al., 2009). Contudo, para alguns táxons de insetos, como as vespas sociais, os estudos sobre a influência da altitude são escassos e sem registros no Brasil (KUMAR, et al., 2009).

Apesar do relevante papel desses insetos nos mais diferentes ecossistemas, não se tem registros de trabalhos publicados sobre a variação nas taxocenoses em gradientes altitudinais no Brasil (SOUZA; ZANUNCIO, 2012).

O conhecimento da distribuição de vespas sociais pode revelar padrões distintos, importante para propostas de políticas de conservação e para a compreensão da distribuição geográfica, bem como a sua diversidade local. O presente trabalho teve por objetivo avaliar a influência da altitude sobre a diversidade e composição das comunidades de vespas sociais, bem como frequência e abundância em fragmentos de florestas estacionais semideciduais em clima tropical de altitude.

\section{Material e Métodos}

O estudo foi conduzido nos municípios de Ouro Fino e Camanducaia, localizados na porção sul do estado de Minas Gerais. A vegetação é do tipo Floresta Estacional Semidecidual Montana. O clima de ambos é do tipo tropical de altitude com verão chuvoso e ameno e período seco no inverno, com temperaturas médias anual variando de $18{ }^{\circ} \mathrm{C}$ a $36{ }^{\circ} \mathrm{C}$ no verão e $5{ }^{\circ} \mathrm{C}$ no inverno no município de Ouro Fino, e médias inferiores, com temperaturas abaixo de zero em Monte Verde, distrito de Camanducaia (IBGE, 2000).

Três localidades de amostragens foram selecionadas: duas cotas altimétricas (Cota 01, entre 800 e 1100 m, e Cota 02 , entre 1300 a $1600 \mathrm{~m}$ ) localizadas no município de Ouro Fino (22 16 '58'S e 46 $22^{\prime}$ '08'O (Figura 1B); e uma (Cota 03, entre 1800 a $2100 \mathrm{~m}$ ) no distrito de Monte Verde, município de Camanducaia (22086’30"S e 4603'89"O) (Figura 1A). As três áreas do estudo apresentavam diferentes tipos de edificações como fazendas, chalés e currais.

O estudo foi conduzido no período de junho de 2013 a março de 2014, com 24 dias de amostragem, sendo oito dias para cada cota altimétrica. As espécies de vespas sociais foram coletadas por meio da metodologia de busca ativa com esforço de campo de quatro pessoas (SOUZA; PREZOTO, 2006). As coletas foram realizadas no intervalo entre 9 e $16 \mathrm{~h}$ de forma aleatória em trilhas abertas ou existentes nas áreas de campo, mata semidecidual, em afloramentos rochosos, áreas de mata ciliar, cavidades em tronco de árvores, vegetação de folhas largas, dossel (com auxílio de binóculo), e termiteiros de montículo.

Os espécimes capturados foram fixados (em via úmida e seca) e transportados para o laboratório de zoologia do Instituto Federal Sul de Minas Campus Inconfidentes, Minas Gerais. As espécies foram identificadas por meio de comparações com exemplares da coleção de vespas sociais do Instituto Federal Sul de Minas, Campus Inconfidentes, MG e de chaves dicotômicas de identificação de gêneros e espécies (RICHARDS, 1978; CARPENTER; MARQUES, 2001). 
FIGURA 1: Áreas amostradas para coleta de vespas sociais no distrito de Monte Verde, município de Camanducaia (A), e no município de Ouro Fino (B) no sul do estado de Minas Gerais

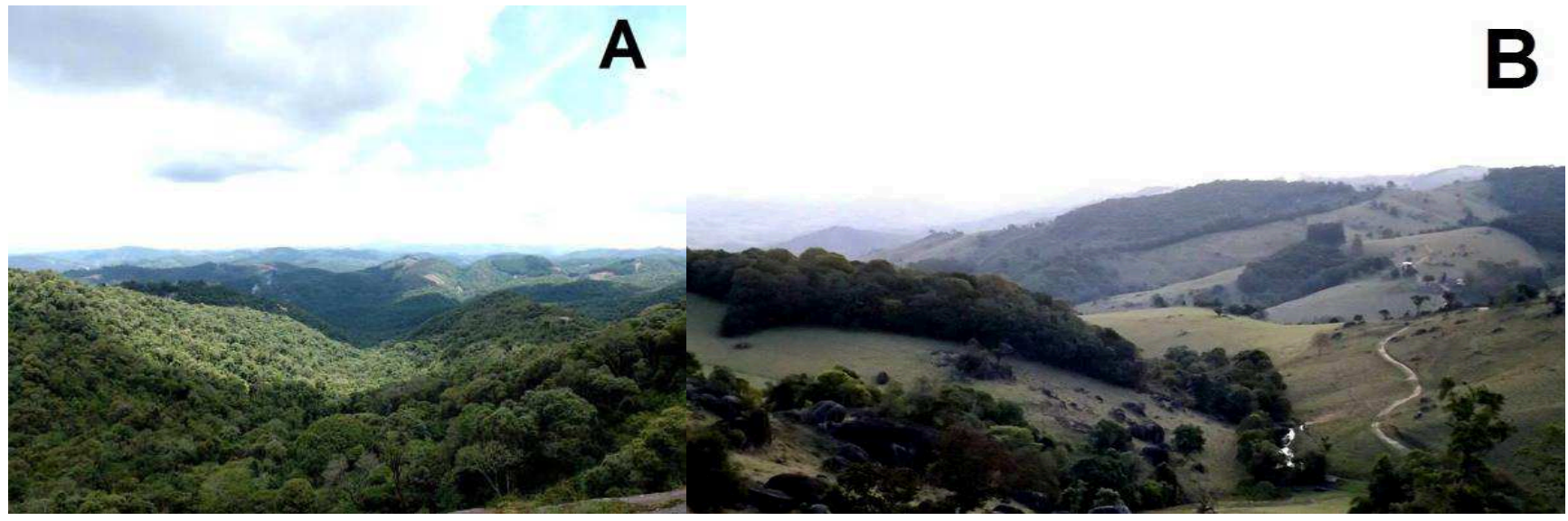

Exemplares de cada morfoespécie foram enviados ao especialista na taxonomia de vespas sociais Orlando Tobias Silveira do Museu Paraense Emílio Goeldi em Belém, PA.

As vespas sociais foram classificadas de acordo com sua frequência de indivíduos em espécies constantes (em $50 \%$ a $100 \%$ das coletas), acessórias ( $25 \%$ a $49 \%$ das coletas) ou acidentais (abaixo de 25\%) (SOUZA; PREZOTO, 2006).

O estimador de riqueza Jackknife 1 calculou a riqueza a partir de dados de uma amostragem e Jackknife 2, duas amostragens (SMITH; PONTIUS, 2006). Essa projeção da riqueza de espécies obtida por esses estimadores de riqueza foi calculado através do programa EstimateS Win 8.0 (COLWELL, 2013).

A comparação entre as faunas de vespas sociais coletada nas três cotas foi feita pela análise de agrupamento (UPGMA) por meio do coeficiente de similaridade de Jaccard (KREBS, 1989), que leva em consideração a ocorrência das espécies em cada área.

\section{Resultados e Discussão}

Foram registradas 34 espécies de vespas sociais distribuídas em 11 gêneros, considerando as três cotas altimétricas. A Cota 01 apresentou maior riqueza especifica $(S=27)$, seguido respectivamente pela Cota $02(\mathrm{~S}=19)$ e Cota $03(\mathrm{~S}=11)$ (Tabela 1$)$.

O maior valor de riqueza de vespas sociais na Cota 01 ocorreu provavelmente em função de características ambientais mais favoráveis, possibilitando uma maior quantidade de recursos alimentares, sítios de nidificação e condições climáticas favoráveis, como temperatura (RICHTER, 2000; ELPINO-CAMPOS et al., 2007; SOUZA et al., 2014).

Com base no coeficiente de similaridade de Jaccard entre as faunas de vespas sociais das três áreas, foi possível observar a formação de dois grupos. Um formado pelas cotas 02 e $03(50 \%)$, e o outro pela cota 01 , a qual apresentou similaridade de $32 \%$ com primeiro grupo (Figura 2).

As espécies Agelaia multipicta, Mischocyttarus cassununga, Polistes simillimus, $P$. fastidiosuscula, P. occidentalis occidentalis, P. paulista, P. scutellaris e Protonectarina sylveirae, foram registradas nas três cotas altimétricas (Tabela 1). Apoica gelida, Brachygastra lecheguana, M. drewseni, M. wagneri e P. ignobilis foram exclusivas das Cotas 01 e 02 . As espécies $P$. minarum e $P$. punctata, foram registradas somente nas Cotas 02 e 03. 
TABELA 1: Lista de espécies de vespas sociais presentes nas diferentes cotas altimétricas nos municípios de Ouro Fino e Camanducaia, Minas Gerais. Cota 01 (C1), entre 800 a $1100 \mathrm{~m}$ de altitude; Cota 02 (C2), entre 1300 a $1600 \mathrm{~m}$; Cota 03 (C3), entre 1800 a $2100 \mathrm{~m} .1$ = presença de invólucro: P = ausência de invólucro: A: $\mathrm{F}=$ Frequência.

\begin{tabular}{|c|c|c|c|c|c|}
\hline & & F \% & F\% & F \% & Invólucro \\
\hline & Espécies & $\mathbf{C} 1$ & C 2 & C 3 & \\
\hline \multirow{20}{*}{ 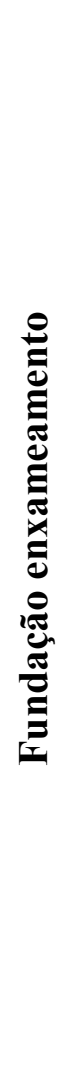 } & Agelaia multipicta (Haliday, 1836) & 75 & 100 & 37,5 & A \\
\hline & Agelaia pallipes (Olivier, 1791) & 12,5 & 0 & 0 & A \\
\hline & Apoica gelida Van der Vecht, 1973 & 12,5 & 62,5 & 0 & A \\
\hline & Brachygastra augusti (Saussure, 1854) & 12,5 & 0 & 0 & $\mathrm{P}$ \\
\hline & Brachygastra lecheguana (Latreille, 1824) & 37,5 & 50 & 0 & $\mathrm{P}$ \\
\hline & Parachartergus fraternus (Griboldo, 1892) & 12,5 & 0 & 0 & $\mathrm{P}$ \\
\hline & Polybia chrysothorax (Lechtenstein, 1796) & 62,5 & 0 & 0 & $\mathrm{P}$ \\
\hline & Polybia fastidiosuscula Saussure, 1854 & 37,5 & 62,5 & 100 & $\mathrm{P}$ \\
\hline & Polybia ignobilis (Haliday, 1836) & 25 & 25 & 0 & $P$ \\
\hline & Polybia jurinei Saussure, 1854 & 12,5 & 0 & 0 & $\mathrm{P}$ \\
\hline & Polybia minarum Ducke, 1906 & 0 & 50 & 38 & $\mathrm{P}$ \\
\hline & Polybia occidentalis occidentalis (Oliver, 1791) & 25 & 12,5 & 88 & $\mathrm{P}$ \\
\hline & Polybia paulista $\mathrm{H}$. Von Ihering 1896 & 75 & 100 & 63 & $\mathrm{P}$ \\
\hline & Polybia punctata du Buysson, 1907 & 0 & 25 & 75 & $\mathrm{P}$ \\
\hline & Polybia scutellaris (White, 1841) & 37,5 & 62,5 & 50 & $\mathrm{P}$ \\
\hline & Polybia sericea (Olivier, 1791) & 0 & 25 & 0 & $\mathrm{P}$ \\
\hline & Protonectarina sylveirae (Saussure, 1854) & 75 & 50 & 50 & $\mathrm{P}$ \\
\hline & Protopolybia sedula (Saussure, 1854) & 0 & 50 & 0 & $\mathrm{P}$ \\
\hline & Pseudopolybia vespiceps (Saussure, 1864) & 25 & 0 & 0 & $\mathrm{P}$ \\
\hline & Synoeca cyanea (Fabricius, 1775) & 37,5 & 0 & 0 & $\mathrm{P}$ \\
\hline \multirow{14}{*}{ 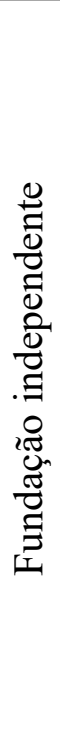 } & Mischocyttarus atramentarius Zikán, 1949 & 0 & 62,5 & 0 & A \\
\hline & Mischocyttarus cassununga (R. Von.Ihering, 1903) & 87,5 & 87,5 & 75 & A \\
\hline & Mischocyttarus cerberus (Richards, 1940) & 50 & 0 & 0 & A \\
\hline & Mischocyttarus drewseni Saussure, 1857 & 12,5 & 87,5 & 0 & A \\
\hline & Mischocyttarus mourei Zikán 1949 & 0 & 0 & 13 & A \\
\hline & Mischocyttarus paraguaensis Zikán, 1935 & 50 & 0 & 0 & A \\
\hline & Mischocyttarus rotundicollis (Cameron,1912) & 0 & 37,5 & 0 & A \\
\hline & Mischocyttarus wagneri (du Buysson, 1908) & 75 & 12,5 & 0 & A \\
\hline & Polistes cinerascens Saussure, 1853 & 25 & 0 & 0 & A \\
\hline & Polistes ferreri Saussure, 1853 & 25 & 0 & 0 & A \\
\hline & Polistes lanio lanio (Fabricius, 1775) & 25 & 0 & 0 & A \\
\hline & Polistes pacificus pacificus Fabricius, 1804 & 12,5 & 0 & 0 & A \\
\hline & Polistes simillimus Zikán, 1951 & 0 & 50 & 100 & A \\
\hline & Polistes versicolor (Olivier, 1971) & 50 & 0 & 0 & A \\
\hline
\end{tabular}


FIGURA 2: Similaridade da fauna de vespas sociais registradas em três diferentes altitudes em Minas Gerais (Cota 01: 800-100m; Cota 02: 1300-1600m e Cota 03: $1800-2100 \mathrm{~m})$.

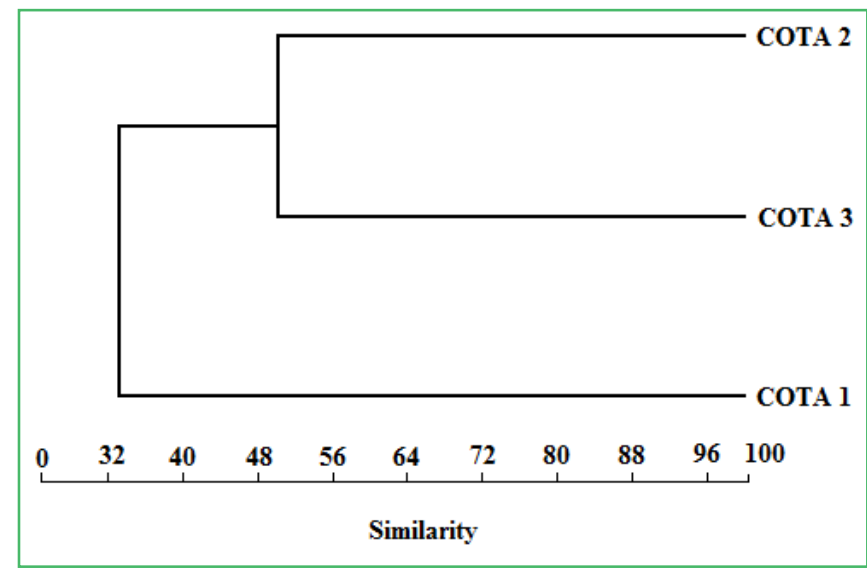

$\mathrm{Na}$ cota 01,14 espécies foram exclusivas, quatro exclusivas a cota 02 , e uma espécie exclusiva para a cota 03, M. mourei, sendo registro inédito para o estado de Minas Gerais. Essa espécie tem ocorrência do Paraná, Santa Catarina e São Paulo (RICHARDS, 1978), sendo que neste estudo, foi registrada apenas uma colônia em substrato vegetal.

O fato de algumas espécies serem comuns em todas as diferentes altitudes, ou restritas a uma determinada cota, pode estar relacionada ao fato de certas espécies de vespas sociais serem euriécias, apresentando ampla valência ecológica, podendo variar seus hábitos de nidificação em função das condições ambientais e substratos de nidificação disponíveis. Outras são consideradas estenoécias, apresentando limites restritos de valência ecológica, ocorrendo apenas em locais com condições especificas (MARQUES; CARVALHO, 1993; SANTOS et al., 2009; SOUZA et al., 2010; 2014; SOUZA; ZANUNCIO, 2012).

O gênero mais frequente nas áreas de estudo foi Polybia, o que corrobora com outros levantamentos realizados em outras regiões de Minas Gerais e do Brasil (DINIZ; KITAYAMA, 1994; SOUZA; PREZOTO, 2006: ELPINO-CAMPOS et al., 2007). Esse fato pode estar relacionado ao número de espécies que compõe o grupo, abundância de indivíduos por colônias e sua distribuição, sendo considerado o gênero mais frequente de vespas sociais na América do Sul (RICHARDS,
1978; MARQUES; CARVALHO, 1993; CARPENTER; MARQUES, 2001).

Considerando a arquitetura dos ninhos, construídos com ou sem invólucro protetor, houve diferença entre as cotas altimétricas. Ninhos com invólucro protetor representaram $44 \%$ das espécies na Cota $01,58 \%$ na Cota 02 e 64\% na Cota 03 (Figura 3). Esses resultados sugerem que espécies que constroem ninhos com invólucro protetor sejam mais frequentes à medida que ocorre uma elevação da altitude em áreas de Floresta Estacional Semidecidual sob influência do clima Tropical de Altitude. A presença do invólucro protetor pode auxiliar na manutenção de uma temperatura favorável a sobrevivência dos indivíduos e desenvolvimento das colônias (HOZUMI et al., 2010).

FIGURA 3: Arquitetura de ninhos de vespas sociais (com ou sem invólucro) distribuídos nas diferentes altitudes amostradas, nos municípios Ouro Fino e Camanducaia, Minas Gerais.

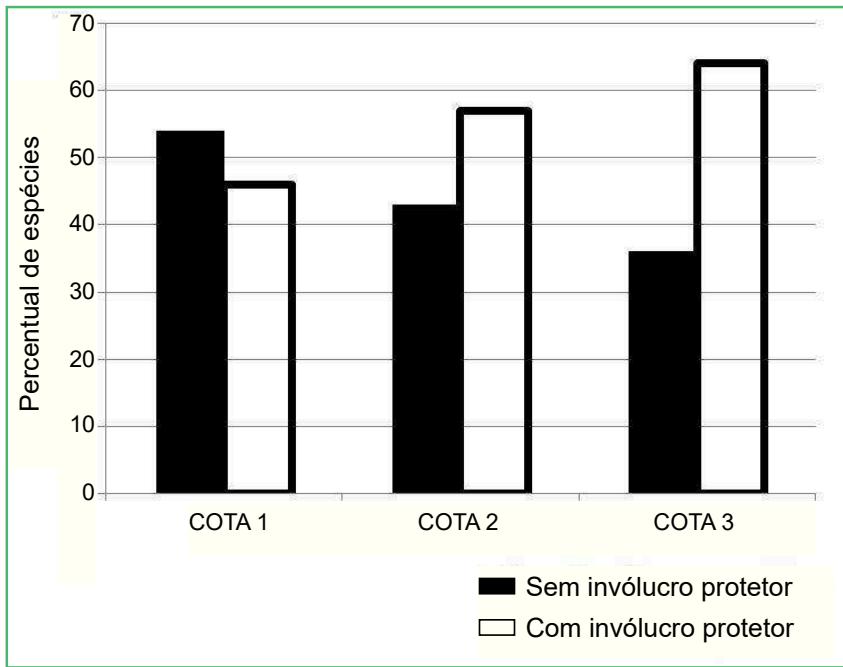

As espécies que apresentam fundação de colônias por enxameamento, como Polybia e Agelaia são mais frequentes em relação aquelas que fundam de forma independente, como Polistes e Mischocyttarus, à medida que se eleve a altitude, o que também foi registrado no estudo de Kumar et al. (2009), realizado na Costa Rica.

Jeanne (1991) apresenta vantagens da fundação de colônias por enxameamento em relação à fundação independente: em primeiro lugar, a mortalidade da rainha é reduzida e essas se encontram melhor protegidas no interior da colônia que possuem invólucro, pois nem 
todas as espécies enxameantes apresentam invólucro, como Apoica e Agelaia; em segundo lugar, permite estabelecimento de colônias mais numerosas. Como os enxames fundadores normalmente têm múltiplas rainhas, a taxa de postura de ovos e crescimento da colônia é rápida em comparação com espécies de fundação independente (WENZEL, 1991; SMITH et al., 2001). Portanto o predomínio de Polybia em altitudes parece ser devido à soma de dois fatores, o padrão arquitetônico do ninho e o tipo de fundação de novas colônias.

Os estimadores de riqueza Jackknife 1 e 2 mostraram que a riqueza na área de estudo na Cota 01 deve ser de 33 a 34 espécies, 23 a 24 espécies para Cota 02, 17 a 18 espécies para Cota 03. Assim, para Cota 01 foi registrado $80 \%$ da riqueza estimada, na Cota 02 foi $88 \%$ e para Cota 03 foi $79 \%$ (Figura 4). A eficiência de coleta nas três cotas altimétricas pode ser considerada satisfatória quando comparado a estudo realizado em área de Floresta Atlântica em Minas Gerais (SOUZA et al., 2012). Esse fato pode ser atribuído à ausência de áreas de vegetação densa, o que facilita a localização dos ninhos e a coleta dos espécimes (SOUZA; PREZOTO, 2006).

FIGURA 4: Curva de acúmulo de espécies de vespas sociais coletadas na Cota 01 - entre 800 a 1100 m de altitude; Cota 02 - entre 1300 a $1600 \mathrm{~m}$; Cota 03 - entre 1800 a $2100 \mathrm{~m}$, em oito dias de amostragem para cada cota em áreas de clima tropical de altitude.

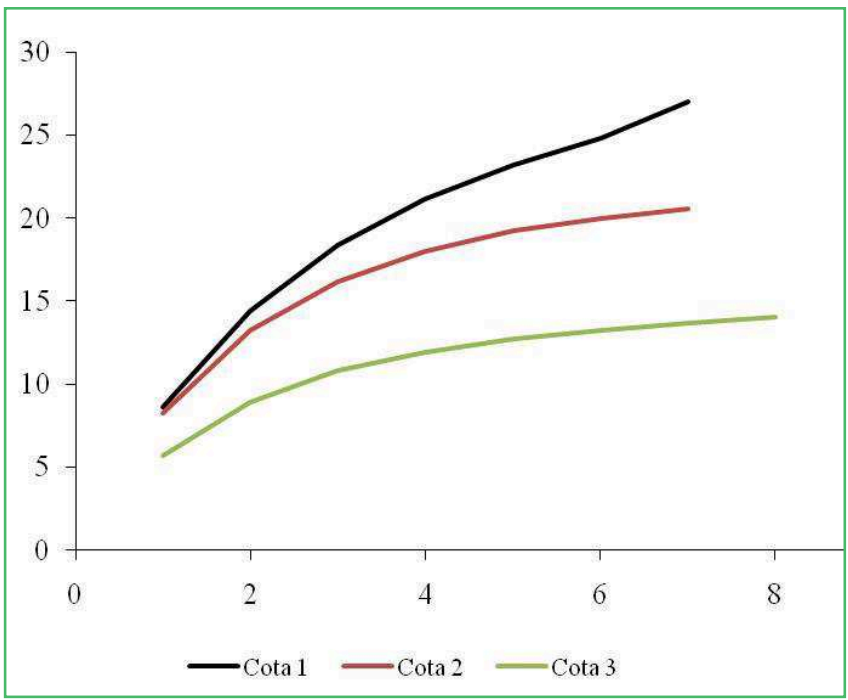

A maior frequência de $M$. cassununga na Cota 01 pode ser explicada pelo fato dessa espécie apresentar um elevado grau de sinatropismo, e também por ser facilmente encontrada em edificações (ALVARENGA et al., 2010). Além disso, a presença de mais de uma fêmea inseminada por colônia, com ovários bem desenvolvidos e o grande sucesso na oviposição, permite uma maior dispersão dessa espécie (MURAKAMI et al., 2009).

A frequência de $A$. multipicta na Cota 02 pode ser devido ao grande número de indivíduos por colônia, comum nesse gênero, pois estes constroem os maiores ninhos, constituindo colônias populosas, tornandoas abundantes nos ambientes onde os ninhos estão localizados, o que aumenta a probabilidade de coleta em diferentes amostragens na mesma área (ZUCCHI et al., 1995; HUNT et al., 2001; HERMES; KÖHLER, 2006).

$\mathrm{Na}$ Cota 03 a alta frequência de $P$. simillimus é justificada pela presença da mesma em edificações antrópicas, o que provavelmente atenua a ausência do invólucro em seus ninhos (SOUZA; PREZOTO, 2006; JACQUES et al., 2012).

As espécies P. paulista, M. cassununga, A. multipicta, $P$. simillimus, $P$. fastidiosuscula, $P$. sylveirae, $P$. scutellaris, foram classificadas como constantes, presente em mais de $50 \%$ das coletas. P. occidentalis, $M$. drewseni, P. punctata, B. lecheguana, M. wagneri, P. minarum e $A$. gelida foram consideradas espécies acessórias, e o restante, que perfaz $65 \%$ das espécies amostradas, foram consideradas acidentais, o que parece ser padrão comum em vespas sociais, como proposto por Souza et al. (2010).

As informações geradas nesse estudo podem ser utilizadas como instrumentos de monitoramento dos ambientes, a fim de acompanhar o reflexo das mudanças climáticas sobre as comunidades de vespas sociais provocadas pelo aquecimento global.

Considerando a importância ecológica das vespas sociais, a ocorrência de espécies em todas as cotas altimétricas, sugere que esse grupo de insetos seja relevante na manutenção das cadeias tróficas ou na 
polinização, mesmo em altitudes acima de $1800 \mathrm{~m}$, o que se desconhecia até o presente momento.

\section{Agradecimentos}

Aos estagiários Filipe Bernardes, Marco Antônio Cunha e Josué Ferreira, pela colaboração nas coletas; ao Hotel "Cabeça de Boi", ao prefeito Rubens Osis e à vereadora Tânia, pela hospedagem em Monte Verde, Camanducaia. Ao professor Dr. Orlando Tobias da Silveira, do Museu Emilio Goeldi, pelo apoio na identificação do material; ao Dr. Jamil de Morais Pereira, pelo auxílio no projeto IFSULDEMINAS. Ao Dr. Ábner Elpino Campos e doutorando Epifânio Porfiro Pires, UFLA, pelas análises estatísticas.

\section{Referências}

ALVARENGA, R. D.; CASTRO, M. M.; SANTOS-PREZOTO, H. H.; PREZOTO, F. Nesting of social wasps (Hymenoptera, Vespidae) in urban gardens in Southeastern Brazil. Sociobiology, Chico, v. 2, n. 55, p. 445-452, 2010.

CARPENTER, J. M.; MARQUES, O. M. Publicações digitais: contribuição ao estudo dos vespídeos do Brasil (Insecta, Hymenoptera, Vespoidea, Vespidae). Vol. 2. Salvador: Universidade Federal da Bahia. 2001. 144 p.

COLWELL, R. K. EstimateS: estimativa estatística da riqueza de espécies e espécies compartilhadas a partir de amostras. Versão 9. Guia do usuário e aplicativo. 2013. Disponivel em: $<$ http://viceroy. eeb.uconn.edu/estimates/>. Acesso em: 18 jan. 2015.

DINIZ, I. R.; KITAYAMA K. Colony densities and preferences for nest habitats of some social wasps in Mato Grosso State, Brazil (Hymenoptera: Vespidae). Journal of Hymenoptera Research, Washington, v. 3, p. 133-143, 1994.

ELPINO-CAMPOS, A.; DEL-CLARO, K.; PREZOTO, F. Diversity of social wasps (Hymenoptera, Vespidae) in cerrado fragments of Uberlândia, Minas Gerais State, Brazil. Neotropical Entomology, Piracicaba, v. 36, p. 685-692, 2007.

FERNANDES, G. W.; LARA, A. C. F. Diversity of Indonesian gallforming herbivores along altitudinal gradients. Biology Letters, London, v. 1, n. 6, p. 186-192, 1993.

FERNANDES, G. W.; PRICE, P. W. Comparisons of tropical and temperate galling species richness: the roles of environmental harshness and plant nutrient status. In: PRICET P. W.; LEWINSOHN, M.; FERNANDES, G. W.; BENSON, W. W. (Ed.). Plant-animal interactions: evolutionary ecology in tropical and temperate regions. New York: John and Sons, Wiley, 1991. p. 91115 .

HENRIQUES-OLIVEIRA, A. L.; NESSIMIAN, J. L. Aquatic macroinvertebrate diversity and composition in streams along an altitudinal gradient in Southeastern Brazil. Biota Neotropica, Campinas, v. 10, n. 3, 2010.

HERMES, M. G.; KÖHLER, A. The flower-visiting social wasps (Hymenoptera, Vespidae, Polistinae) in two areas of Rio Grande do Sul state, southern Brazil. Revista Brasileira de Entomologia, Curitiba, v. 50, n. 2, p. 268-274, 2006.

HOZUMI, S.; MATEUS, S.; KUDÔ, K.; KUWAHARA, T.; YAMANE, S.; ZUCCHI, R. Nest Thermoregulation in Polybia scutellaris (White) (Hymenoptera: Vespidae). Neotropical Entomology, Piracicaba, v. 39, n. 5, p. 826-828, 2010.

HUNT, J. H.; O’DONNELLl, S.; CHERNOFF, N.; BROWNIE, C. Observations on two neotropical swarn-founding wasps Agelaia yepocapa and Agelaia panamaensis (Hymenoptera: Vespidae). Annals of the Entomological Society of American, Annapolis, v. 94, n. 4, p. 555-562, 2001.

IBGE - INSTITUTO BRASILEIRO DE GEOGRAFIA E ESTATÍSTICA. Censo 2000. 2000. Disponível em: $<$ http://www. ibge.gov.br/censo/>. Acesso em: 22 fev. 2015.

JACQUES, G. C.; SOUZA, M. M.; ZANUNCIO, J. C. Diversity of social wasps in the Campus of the Universidade Federal de Viçosa in Viçosa, Minas Gerais State, Brazil. Sociobiology, Chico, v. 59, n. 3, p. 1053-1063, 2012.

JANZEN, D. H. Sweep samples of tropical foliage insects: effects of seasons, vegetation types, elevations, time of day, and insularity. Ecology, New York, v. 54, n. 3, 687-708, 1973.

JEANNE, R. L. The swarm founding Polistinae. In: ROSS, K. G.; MATTHEWS, R. W. (Ed). The social biology of wasps. Ithaca: Cornell University Press, p. 191-231, 1991.

KREBS, C. J. Ecological metodology. New York: Harper \& Hall, 1989. 654 p.

KUMAR, A.; LONGINO, J. T.; COLWELL, R. K.; O'DONNELL, S. Elevational patterns of diversity and abundance of eusocial paper wasps (Vespidae) in Costa Rica. Biotropica, Gainesville, v. 41, n. 3, 338-346, 2009.

LAWTON, J. H.; MACOARVIN, M.; HEADS, P. A. Effects of altitude on the abundance and species richness of insect herbivores on bracken. Journal of Animal Ecology, London, v. 56, p. 147160, 1987.

MARQUES, O. M.; CARVALHO, C. A. L. Hábitos de nidificação de vespas sociais (Hymenoptera: Vespidae) o município de Cruz das Almas, estado da Bahia. Insecta, Cruz das Almas, v. 2, n. 2, p. 23-40, 1993.

MURAKAMI, A. S. N.; SHIMA, S. N; DESUÓ, I. C. More than one inseminated female in colonies of the independent-founding wasp Mischocyttarus cassununga von Ihering (Hymenoptera, Vespidae). Revista Brasileira de Entomologia, Curitiba, v. 53, n. 4, 653-662, 2009.

O'DONNELL, S. Eusocial wasps (Vespidae: Polistinae). In: NADKARNI, N. M.; WHEELWRIGHT, N. T. (Ed.). Monteverde: ecology and conservation of a tropical cloud forest. 1 ed. Oxford: Oxford University Press, 2000. p. 129-131.

RICHARDS, O. W. The social wasps of the Americas. London: British Museum, 1978. 580 p. RICHTER, M. R. Social wasp (Hymenoptera: Vespidae) foraging behavior. Annual Review of Entomology, Palo Alto, v. 45, n. 1, p. 121-150, 2000. 
SANDERS, N. J. Elevational gradients in ant species richness: area, geometry, and rapoport's rule. Ecography, Lund, v. 25, p. 2532,2002

SANTOS, G. M. M.; BISPO, P. C.; AGUIAR, C. M. L. Fluctuations in richness and abundance of social wasps during the dry and wet seasons in three phyto-physiognomies at the tropical dry forest of Brazil. Environmental Entomology, Lanham, v. 38, n. 6, p. 16131617, 2009.

SMITH, A. R.; O'DONNELL, S.; JEANNE, R. L. Correlated evolution of colony defense and social structure: A comparative analysis in eusocial wasps (Hymenoptera: Vespidae). Evolutionary Ecology Research, Tucson, v. 3, p. 331-344, 2001.

SMITH, C.D.; PONTIUS, J.S. Jackknife Estimator of Species Richness with S-PLUS. Journal of Statistical Software, Linz, v. 15, p. 1-12, 2006.

SOUZA, M. M.; LADEIRA, T. E.; ASSIS, N. R. G.; ELPINOCAMPOS, A.; CARVALHO, P.; LOUZADA, J. Ecologia de vespas sociais (Hymenoptera, Vespidae) no Campo Rupestre na Área de Proteção Ambiental, APA, São José, Tiradentes, MG. MG-BIOTA, Belo Horizonte, MG, v. 3, n. 2, p. 1-30, 2010.

SOUZA, M. M.; PIRES, E. P.; FERREIRA, M.; LADEIRA, T. E.; PEREIRA, M.; ELPINO-CAMPOS, A.; ZANUNCIO, J. C. Biodiversidade de vespas sociais (Hymenoptera: Vespidae) do Parque Estadual do Rio Doce, Minas Gerais, Brasil. MG. Biota, Belo Horizonte, v. 5, p. 4-20, 2012.
SOUZA, M. M.; PIRES, P.; PREZOTO, F. Seasinal richness and composition of social wasps (Hymenoptera, Vespidae) in areas of cerrado biome in Barroso Minas Gerais, Brasil. Bioscience Journal, Uberlândia, v. 30, n. 2, p. 539-545, 2014.

SOUZA, M. M.; PREZOTO, F. Diversity of social wasps (Hymenoptera, Vespidae) in Semideciduous forest and cerrado (savanna) regions in Brazil. Sociobiology, Chico, v. 47, n. 1, p. 135-147, 2006.

SOUZA, M. M.; ZANUNCIO, J. C. Marimbondos: vespas sociais (Hymenoptera: Vespidae). Viçosa: Editora UFV, 2012. 79 p.

WENZEL, J. W. Evolution of nest architecture in social vespids. In: KROSS, G.; MATTHEWS, R. W. (Ed). The social biology of wasps. Ithaca: Cornell University Press, 1991. p. 480-519.

WOLDA, H. Altitude, habitat and tropical insect diversity. Biological Journal of the Linnean Society, London, v. 30, p. 313323, 1987.

ZUCCHI, R.; SAKAGAMI, S. F.; NOLL, F. B.; MECHI, M. R.; MATEUS, S.; BAIO, M. V.; SHIMA, S. N. Agelaia vicina, a swarm-founding Polistine with the largest colony size among wasps and bees (Hymenoptera: Vespidae). Journal of the New York Entomological Society, New York, v. 103, n. 2, p. 129-137, 1995. 